\title{
Impacts of Parental Burnout on Chinese Youth's Mental Health: The Role of Parents' Autonomy Support and Emotion Regulation
}

\author{
Beiming Yang ${ }^{1} \cdot$ Bin-Bin $\mathrm{Chen}^{2} \cdot$ Yang Qu $\mathbb{1}^{1} \cdot$ Yuanfei Zhu ${ }^{3}$
}

Received: 23 February 2021 / Accepted: 5 May 2021 / Published online: 9 June 2021

(c) The Author(s), under exclusive licence to Springer Science+Business Media, LLC, part of Springer Nature 2021

\begin{abstract}
Parental burnout is a state that parents experience overwhelming exhaustion in their parental role. Given the detrimental impacts of parents' stress on adolescent development, youth may suffer from undesirable emotional adjustment due to parental burnout. Therefore, it is key to understand the underlying mechanisms through which parental burnout may play a role in youth's mental health and identify protective factors that may reduce the potential negative impacts. Using a sample of 442 Chinese parent-adolescent dyads (Mean age of youth $=13.35$ years; $50 \%$ girls), this two-wave longitudinal study examined how parental burnout contributes to youth's mental health over the span of two months. Moreover, the current research investigated the potential mediating role of autonomy support and the potential moderating role of emotion regulation in the links between parental burnout and youth's mental health. The results showed that greater parental burnout was predictive of youth's greater depressive and anxiety symptoms two months later, and such effects were partially mediated by less autonomy-supportive parenting. Notably, the negative effects of parental burnout on autonomy-supportive parenting and youth's mental health were not significant when parents used more cognitive reappraisal to regulate their emotions. These findings demonstrate the underlying mechanisms of how parental burnout affects youth's mental health over time and highlight the protective role of healthy emotion regulation against parental burnout.
\end{abstract}

Keywords Adolescents $\cdot$ Autonomy support $\cdot$ Emotion regulation $\cdot$ Mental health $\cdot$ Parental burnout

\section{Introduction}

Under the influence of enduring parenting stress, parents may feel exhausted in taking care of their children. This parenting experience, namely parental burnout, is a chronic condition characterized by feeling overwhelmed due to one's parental role and emotional distancing from one's children (Mikolajczak et al., 2019). In recent years, parental burnout has begun to receive increasing scholarly attention (Roskam et al., 2018). For example, prior studies have investigated correlates of parental burnout from multiple facets (i.e., sociodemographic, child-related, parent-related, and family-functioning factors) to investigate the potential

These authors contributed equally: Beiming Yang, Bin-Bin Chen

Bin-Bin Chen

chenbinbin@fudan.edu.cn

Yang Qu

yangqu@northwestern.edu causes of such burnout (e.g., Furutani et al., 2020; Mikolajczak et al., 2018). However, very little is known about the impacts of parental burnout on adolescent development. Moreover, no prior research has examined protective factors against parental burnout or underlying mechanisms through which parental burnout may affect adolescent adjustment. Given that adolescence is a developmental stage marked by the onset of several emotional problems (Lee et al., 2014), it is highly needed to conduct empirical research, and especially longitudinal research, to examine the role of parental burnout in youth's mental health. To address this gap, the current study employed a longitudinal design to investigate the effects of parental burnout on youth's depressive and anxiety symptoms, with attention to the mediating role of autonomy-supportive parenting and the moderating role of parents' emotion regulation.

\footnotetext{
Northwestern University, Evanston, USA

Fudan University, Shanghai, China

East China Normal University, Shanghai, China
} 


\section{Parental Burnout and Youth's Mental Health}

Parental burnout may be detrimental to youth's mental health, contributing to youth's emotional maladjustment over time. Past studies have consistently shown that various parental mental health problems (e.g., distress, depression, and anxiety) are linked with youth's dampened psychological well-being (Brennan et al., 2002; Frasquilho et al., 2016; Pereira et al., 2014). Moreover, parenting stress has long been considered as a source of children's mental health problems (Deater-Deckard, 1998). Longitudinal research suggests that parents' high stress level is predictive of youth's mental health problems such as depression and anxiety over time (Bakoula et al., 2009). Because burnout is mostly resulted from excessive and prolonged exposure to stress (Leiter et al., 2014; Maslach \& Leiter, 2016), parental burnout may also have a negative impact on youth's mental health. Indeed, a recent study indicates a positive concurrent correlation between parental burnout and youth's loneliness (Cheng et al., 2020). However, no extant research has examined the longitudinal effects of parental burnout on youth's mental health.

\section{Autonomy Support as a Potential Mechanism}

Autonomy-support parenting (i.e., parents' willingness to consider children's perspectives and allow children to make decisions) may play a mediating role in the effects of parental burnout on youth's mental health. A few studies have found concurrent and longitudinal associations between parental burnout and negative parenting practices (e.g., parental neglect and violence; Mikolajczak et al., 2018; Mikolajczak et al., 2019). Regarding autonomy support, past studies suggest that parents tend to become less autonomy-supportive when they are under stress (Grolnick et al., 2002; Wuyts et al., 2017). In this case, granting children autonomy support may be particularly effortful and challenging for parents under stress. As for parental burnout, when parents feel exhausted and overwhelmed, they may not have the energy to consider youth's opinions. Therefore, parents who experience parental burnout may be less likely to grant their youth autonomy support.

Subsequently, less autonomy support may result in youth's dampened mental health over time. According to self-determination theory, the need for autonomy is one of the three basic psychological needs (i.e., competence, autonomy, and relatedness) that are essential for individuals' well-being across cultures (Ryan \& Deci, 2000, 2017). From a self-determination theory perspective, individuals may suffer emotionally in environments that lack autonomy support, because they may feel a loss of volition, will, and choice (Ryan \& Deci, 2006). Therefore, less autonomy-supportive parenting may not meet youth's need for autonomy, and thus it may induce ill-being including mental health problems (Soenens et al., 2007; Soenens et al., 2015). Moreover, adolescence is a period that children increasingly seek to individuate from their parents (Koepke \& Denissen, 2012). In this case, autonomy support could be particularly important for youth's psychological well-being, because it fosters youth's individuality (Barber et al., 2005). Indeed, cross-sectional and longitudinal studies have shown that a lack of parental autonomy support predicts youth's dampened psychological well-being (Marbell-Pierre et al., 2019; van der KaapDeeder et al., 2017; Wang et al., 2007). Taken together, parental burnout may contribute to youth's mental health problems through less autonomy-supportive parenting.

\section{Emotion Regulation Considerations}

Parents' emotion regulation may buffer the negative effects of parental burnout on youth's mental health. Prior studies with different methods (e.g., self-reported survey, biological assessment, and neuroimaging) have consistently shown that effective emotion regulation can be a protective factor against stress (Kao et al., 2019; Shahane et al., 2019; Speer $\&$ Delgado, 2017). The use of cognitive reappraisal (i.e., reinterpreting emotion-eliciting situations to change one's subjective evaluations of the event) and expressive suppression (i.e., inhibiting the outward expression of one's emotions) are two common strategies to regulate negative emotions (Gross \& John, 2003). Compared to expressive suppression, the use of cognitive reappraisal is generally more effective in regulating negative emotions and has positive impacts on well-being (for reviews, see Dryman \& Heimberg, 2018; John \& Gross, 2004). For example, past research suggests that the use of cognitive reappraisal, but not expressive suppression, is effective in conferring resilience to stress (Johnson et al., 2016). With regard to parental burnout, the extent to which parents can effectively regulate their negative emotions may moderate the negative impacts of such burnout. For parents who can effectively regulate their emotions in the face of burnout, they may engage in more positive parenting practices when they interact with their youth despite the burnout. In this case, parents' distress and negative emotions may not transmit to their youth. Therefore, parental burnout may not lead to youth's mental health problems among parents who can effectively regulate their negative emotions.

Regarding the potential mediating role of autonomysupportive parenting in linking parental burnout and youth's mental health, emotion regulation may also moderate this mediation path, such that the undesirable impact of parental burnout on parenting practices may be buffered by parents' effective emotion regulation. Effective emotion regulation tends to reduce the negative effects of parental mood 


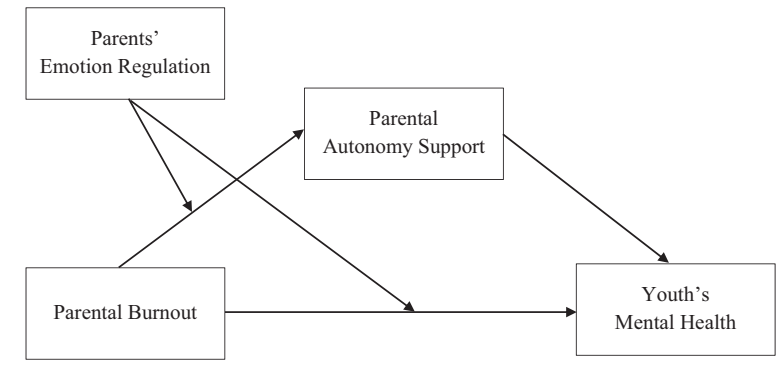

Fig. 1 The conceptual moderated mediation model for parental autonomy support and parents' emotion regulation in the links between parental burnout and youth's mental health

disorders on parenting practices and children's socioemotional functioning (for a review, see Rutherford et al., 2015). For example, prior research suggests that maternal stressors (e.g., being a single parent, number of children in the home, and household chaos) are associated with mothers' negative parenting behaviors and worse parentchild relationships only among mothers who have poor emotion regulation (Deater-Deckard et al., 2016). Therefore, parental burnout may only lead to less autonomysupportive parenting when parents have poor emotion regulation skills. As shown in Fig. 1, a moderated mediating model was constructed to clarify the mechanism - the mediating role of autonomy support and the moderating role of emotion regulation in the links between parental burnout and youth's mental health.

\section{Current Study}

The current study was guided by three goals. The first goal was to examine whether parental burnout is predictive of youth's mental health over time. It was hypothesized that parental burnout has longitudinal effects on youth's greater depressive and anxiety symptoms. The second goal was to explore the potential mechanisms underlying the effects of parental burnout on youth's mental health. It was anticipated that greater parental burnout may be related to less autonomy-supportive parenting, leading youth to experience more depressive and anxiety symptoms over time. The third goal was to investigate the potential moderating role of parents' emotion regulation in the links between parental burnout and youth's mental health. Two types of emotion regulation strategies (i.e., cognitive reappraisal and expressive suppression) were examined in the current study. It was hypothesized that parental burnout is only predictive of youth's mental health problems when parents are less capable of regulating their emotions. Moreover, it was anticipated that effective emotion regulation (i.e., the use of cognitive reappraisal) also moderates the associations between parental burnout and autonomy support, such that parental burnout only associates with less autonomysupportive parenting when parents exhibit a lower level of emotion regulation.

To test these hypotheses, the current research employed a two-wave longitudinal design and studied Chinese parentadolescent dyads. At Wave 1, parents reported on their parental burnout and their use of cognitive reappraisal and expressive suppression to regulate negative emotions. Moreover, parental autonomy support was assessed at Wave 1. At each wave, youth reported on their depressive and anxiety symptoms.

\section{Methods}

\section{Participants}

The sample consisted of 442 Chinese parent-adolescent dyads. Youth were seventh-graders (Mean age $=13.35$ years, $S D=0.36$ years) and were evenly distributed across sex $(50 \%$ girls). Youth were recruited from three middle schools in Shanghai. One school was above-average and the other two were average in terms of achievement, with families primarily from working- and middle-class backgrounds. Parents who participated in the current study were primary caregivers of the youth (70\% mothers and 30\% fathers; Mean age $=41.80$ years; $S D=3.81$ years). Regarding family backgrounds, $79 \%$ of families were twoparent biological families and $70 \%$ of children were the only child in the family. With regard to mothers' educational attainment, 33\% did not complete high school, $20 \%$ had a high school degree, and $47 \%$ had education beyond high school (e.g., a bachelor's or master's degree). With regard to fathers' educational attainment, $29 \%$ did not complete high school, 19\% had a high school degree, and $52 \%$ had education beyond high school (e.g., a bachelor's or master's degree).

\section{Procedure}

Longitudinal data from youth and their parents were collected twice via online questionnaires over two months. The current research was conducted during the time when the region was under significant influence of COVID-19 (Wave 1: early July 2020; Wave 2: early September 2020), such that COVID-19 specific health behavior (e.g., wearing a mask) was mandatory in public places and the health code system (i.e., a color-based tracking system that tracks all people's movement) was in place to control the spread of COVID-19. Among the 442 families who participated at Wave 1, 376 of them continued to participate at Wave 2 . Attrition from Wave 1 to Wave 2 was $14.9 \%$. Comparison of participants completing both waves to those completing 
only the first revealed no differences at Wave 1 on any of the variables examined in this report, $F \mathrm{~s}<3.93, p \mathrm{~s}>0.53$. Moreover, results in Little's MCAR test $\left(\chi^{2}=3.178\right.$, $p=0.79$ ) suggested that missing cases were likely to be missing completely at random (MCAR; Little, 1988). To handle missing data, analyses were conducted with MPlus 8.2 (Muthén \& Muthén, 2017), which uses Full Information Maximum Likelihood (FIML) estimation to provide reliable standard errors under a wide range of conditions. At each wave, extensive explanations of the research were given, and participants completed the online consent before taking the questionnaire. Ethical approval for the study was obtained from the Institutional Review Board in the School of Social Development and Public Policy at Fudan University. Families received small gifts for their participation.

\section{Measures}

\section{Parental burnout}

At Wave 1, parental burnout was assessed using the Parental Burnout Assessment (PBA; Roskam et al., 2018), which has been used to measure parental burnout across countries (e.g., Mikolajczak et al., 2019; Roskam et al., 2021; Sorkkila \& Aunola, 2020). The Chinese version of this measure has been validated and showed good reliability (Cheng et al., 2020). On a 7-point Likert scale $(0=$ never, $6=$ daily), parents reported on the frequency of their feelings of parental burnout such as exhaustion and irritation related to parenting (23 items; e.g., "I feel completely run down by my role as a parent" and "I cannot take being a parent anymore"). The sum score of all 23 items ranged from 0 to 138. The mean was taken across items, with higher numbers indicating greater parental burnout $(\alpha=0.97)$.

\section{Autonomy-supportive parenting}

At Wave 1, both youth and parents reported on autonomysupportive parenting, which was assessed with a six-item measure adapted from prior research (McPartland \& Epstein, 1977; Robbins, 1994; Steinberg et al., 1992). This measure has been used to examine parental autonomy support across cultures (e.g., Cheung et al., 2016; Wang et al., 2007). Youth reported on how often their parents are autonomy-supportive (e.g., "My parents allow me to make choices for myself whenever possible" and "When my parents want me to do something, they explain why") on a 5 -point Likert scale $(1=$ never, $5=$ very often $)$, and parents responded to the parallel items (e.g., "I allow my child to make choices for himself/herself whenever possible" and "When I want my child to do something, I explain why") on the same scale. The mean across the items was taken $(\alpha \mathrm{s}=$ 0.93 for youth's reports and 0.90 for parents' reports). In analyses, youth-reported and parent-reported parental autonomy support were examined separately.

\section{Parents' emotion regulation strategies}

At Wave 1, parents' emotion regulation strategies were examined in terms of their use of cognitive reappraisal and expressive suppression, assessed using the Emotion Regulation Questionnaire (ERQ, Gross \& John, 2003). The ERQ has been widely used in Chinese populations and showed good reliability (e.g., Soto et al., 2011; Qu et al., 2020). Regarding cognitive reappraisal, parents rated how true $(1=$ not at all true, $7=$ very true) each of the six reappraisal items (e.g., "I control my emotions by changing the way I think about the situation I am in" and "When I want to feel less negative emotion, I change the way I'm thinking about the situation") was of them. The mean was taken across the items, with higher numbers indicating greater used of cognitive reappraisal to regulate emotions $(\alpha=0.90)$. Regarding expressive suppression, parents rated how true $(1=$ not at all true, $7=$ very true) each of the four suppression items (e.g., "I control my emotions by not expressing them" and "I keep my emotions to myself") was of them. The items were averaged, with higher numbers indicating greater use of expressive suppression to regulate emotions $(\alpha=0.80)$.

\section{Youth's depressive symptoms}

At both Wave 1 and Wave 2, youth reported on their depressive symptoms using the Short Mood and Feelings Questionnaire (SMFQ; Angold et al., 1995), a measure that has been widely used to assess youth's depressive symptoms across cultures (Cheung et al., 2016; Orben \& Przybylski, 2019). On a 5 -point Likert scale $(1=$ never, $5=$ very often), youth reported on how they felt or acted (13 items; e.g., "felt miserable or unhappy" and "cried a lot") during the past two weeks. The mean was taken across all items, with higher numbers indicating greater depressive symptoms ( $\alpha=0.95$ at Wave 1 and 0.96 at Wave 2$)$.

\section{Youth's anxiety symptoms}

At both waves, youth reported on their anxiety symptoms using the Generalized Anxiety Disorder-7 scale (GAD-7; Spitzer et al., 2006). The Chinese version of GAD-7 has been validated and has shown good reliability in prior research (Wang et al., 2018). On a 4-point Likert scale $(1=$ not at all, $4=$ nearly every day), youth reported on how often they were bothered by each problem (seven items; e.g., "feeling nervous, anxious, or on edge" and "becoming easily annoyed or irritable") during the past two weeks. The items were averaged, with higher numbers indicating greater anxiety symptoms ( $\alpha \mathrm{s}=0.96$ at both waves). 


\section{Results}

\section{Overview of Analyses}

The current research consisted of three sets of analyses. The first set of analyses examined the effects of parental burnout on youth's mental health over time, such that youth's mental health at Wave 2 was predicted by parental burnout at Wave 1, controlling for youth's prior mental health and other covariates (i.e., youth's age and gender, parents' gender, educational attainment, and birthplace, family type, and number of children). The second set of analyses tested the mediating role of autonomy-supportive parenting (i.e., youth-reported and parent-reported) in the associations between parental burnout and youth's mental health. The third set of analyses examined the moderating role of parents' emotion regulation in the links between parental burnout and parental autonomy support as well as youth's mental health. Two types of emotion regulation strategies (i.e., cognitive reappraisal and expressive suppression) were examined.

\section{Descriptive Analyses}

Table 1 shows descriptive statistics and the Pearson correlations among variables. Parents who reported more parental burnout showed less autonomy support. In families that parents reported more parental burnout, youth showed more depressive symptoms at both waves and more anxiety symptoms at Wave 2 but not Wave 1. In families that youth and parents reported more autonomy-supportive parenting, youth showed fewer depressive and anxiety symptoms at both waves. As for parents' emotion regulation strategies, cognitive reappraisal, but not expressive suppression, was associated with youth's fewer depressive and anxiety symptoms at both waves. Parents' educational attainment was positively related to their autonomy-supportive parenting and cognitive reappraisal, and negatively related to youth's depressive symptoms at both waves. Fathers (vs. mothers) reported less use of expressive suppression but not cognitive reappraisal. Parents of older youth reported more parental burnout, and older youth reported more depressive symptoms at Wave 2 but not at Wave 1. Youth in twoparent biological families reported fewer depressive symptoms at Wave 2 but not at Wave 1. Boys and girls did not show any difference in the variables included in the current study.

\section{Parental Burnout and Youth's Mental Health}

The first set of main analyses was to examine whether parental burnout was predictive of youth's mental health problems over time. To this end, multiple regression models were conducted, in which youth's depressive and anxiety symptoms at Wave 2 was predicted by parental burnout at Wave 1, while controlling for youth's prior depressive /anxiety symptoms and other covariates (i.e., youth's age and gender, parents' gender, educational attainment, and birthplace, family type, and number of children). Results indicated that greater parental burnout at Wave 1 predicted youth's greater depressive and anxiety symptoms at Wave 2 (depressive symptoms: $\beta=0.13, p<0.01$; anxiety symptoms: $\beta=0.18, p<0.001$ ), controlling for youth's prior depressive/anxiety symptoms and other covariates.

\section{The Mediating Role of Autonomy Support in the Links between Parental Burnout and Youth's Mental Health}

The second set of analyses was to test if the relationships among parental burnout and youth's mental health are mediated by youth-reported and/or parent-reported autonomy-supportive parenting. To this end, youth's mental health at Wave 2 was predicted from parental burnout at Wave 1 controlling for youth's prior depressive/anxiety symptoms and other covariates in the context of structural equation modeling (SEM). Notably, the indirect effect from parental burnout at Wave 1 to autonomy-supportive parenting at Wave 1 to youth's mental health at Wave 2 was included in the models. Youth-reported and parent-reported autonomy support were examined separately in the models.

As shown in the Panel A of Fig. 2, youth-reported autonomy support mediated the effects of parental burnout on youth's depressive symptoms over time. Based on 5000 bootstrap resamples, the indirect path from parental burnout to autonomy-supportive parenting to youth's depressive symptoms was significant (indirect effect $=0.04,95 \% \mathrm{CI}$ : [0.01, 0.08]), with a reduction of $30 \%$ in the total effect such that the direct effect was not significant. As shown in the Panel B of Fig. 2, youth-reported autonomy support partially mediated the effects of parental burnout on youth's anxiety symptoms over time. Based on 5000 bootstrap resamples, the indirect path from parental burnout to autonomy-supportive parenting to youth's anxiety symptoms was significant (indirect effect $=0.03,95 \% \mathrm{CI}$ : $[0.01$, $0.06]$ ), with a reduction of $17 \%$ in the total effect. Similarly, parent-reported autonomy support also mediated the effects of parental burnout on youth's depressive and anxiety symptoms (depressive symptoms: indirect effect $=0.02$, 95\% CI: [0.01, 0.03]; anxiety symptoms: indirect effect $=$ $0.03,95 \% \mathrm{CI}:[0.01,0.06])$, with reductions of more than $17 \%$ in the total effects. When youth-reported and parentreported autonomy support were included simultaneously in the models as mediators, only youth-reported autonomy support mediated the effects of parental burnout on youth's depressive and anxiety symptoms (depressive symptoms: 


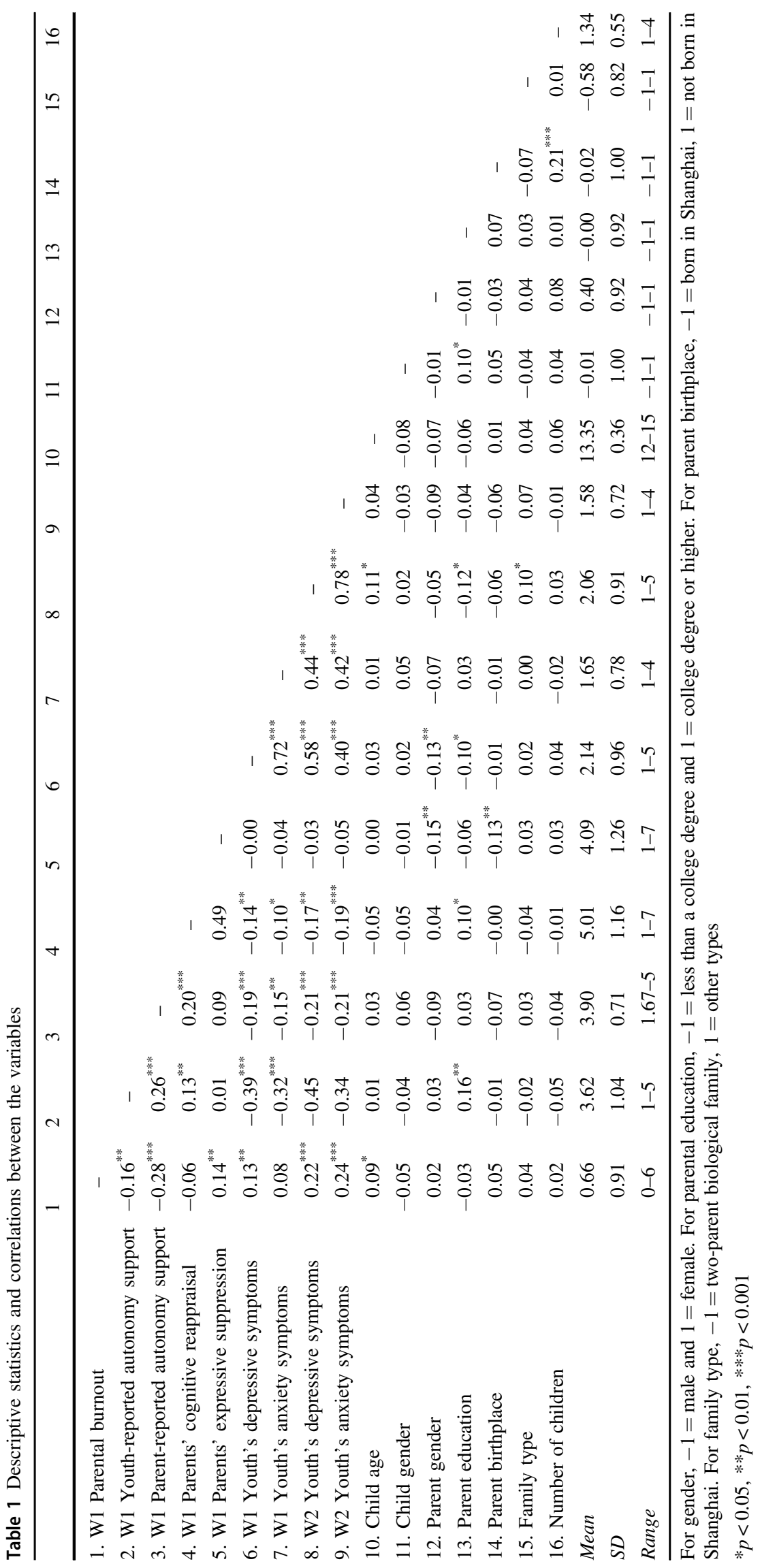



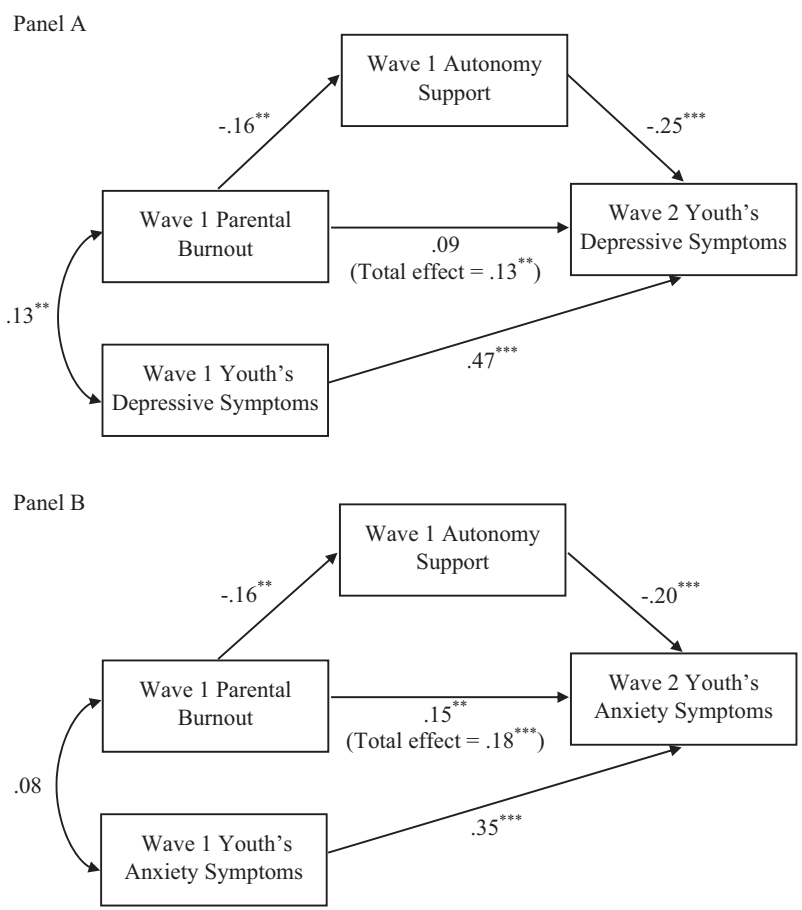

Fig. 2 Autonomy support mediated the effects of parental burnout on youth's depressive symptoms (Panel A) and anxiety (Panel B). Note. Results with youth-reported autonomy support were presented in the figure. Other covariates were included in the models but are not shown for ease of presentation. Standardized coefficients are presented. $* * p<0.01, * * * p<0.001$

indirect effect $=0.04,95 \%$ CI: $[0.01,0.08]$; anxiety symptoms: indirect effect $=0.03$, 95\% CI: $[0.01,0.06]$ ). Taken together, parents who experience greater parental burnout tend to use less autonomy support in their parenting practices, which was associated with more depressive and anxiety symptoms among youth over time.

\section{The Moderating Role of Parents' Emotion Regulation in the Links between Parental Burnout and Youth's Mental Health}

The third set of analyses was to investigate whether parents' two types of emotion regulation strategies (i.e., cognitive reappraisal and expressive suppression) moderate the links between parental burnout and youth's mental health. To this end, regression models were conducted, in which youth's depressive and anxiety symptoms at Wave 2 was predicted by parental burnout, emotion regulation strategy, and the interaction term of parental burnout and emotion regulation strategy at Wave 1, while controlling for youth's prior depressive/anxiety symptoms and other covariates. As shown in the Model 1 of Table 2, parents' cognitive reappraisal moderated the longitudinal effects of parental burnout on youth's depressive and anxiety symptoms
( $p$ s < 0.05). In contrast, as shown in the Model 2 of Table 2, parents' expressive suppression did not moderate the longitudinal links between parental burnout and youth's depressive or anxiety symptoms. Then, the simple slopes of the longitudinal associations between parental burnout and youth's mental health for parents with low (i.e., $1 \mathrm{SD}$ below the mean) and high (i.e., $1 \mathrm{SD}$ above the mean) cognitive reappraisal were plotted. As shown in Fig. 3, in families that parents reported low cognitive reappraisal, greater parental burnout was predictive of youth's more depressive and anxiety symptoms over time (unstandardized simple slopes $>0.25, p s<0.001)$. However, in families that parents reported high cognitive reappraisal, such effects were nonsignificant.

Given that parents' cognitive reappraisal moderated the effects of parental burnout on youth's mental health, the final set of analyses was to examine whether cognitive reappraisal also moderates the associations between parental burnout and autonomy-supportive parenting. To probe this effect, the regression model was conducted to predict autonomy support using parental burnout, emotion regulation strategy, and the interaction term of parental burnout and emotion regulation, controlling for the covariates. Youth-reported and parent-reported autonomy support were examined separately. Regression results indicated that parents' cognitive reappraisal did not moderate the associations between parental burnout and youth-reported autonomysupportive parenting $(t=1.50, p=0.13)$, but moderated the associations between parental burnout and parent-reported autonomy-supportive parenting $(t=3.39, p=0.001)$. Subsequently, the simple slopes of the associations between parental burnout and parent-reported autonomy-supportive parenting for parents with low (i.e., $1 \mathrm{SD}$ below the mean) and high (i.e., $1 \mathrm{SD}$ above the mean) cognitive reappraisal were plotted. As shown in Fig. 4, in families that parents reported low cognitive reappraisal, greater parental burnout was associated with less autonomy-supportive parenting (unstandardized simple slope $=-0.35, p<0.001$ ). However, in families that parents reported high cognitive reappraisal, such effects were non-significant.

\section{Sensitivity Analyses}

Supplemental sensitivity analyses were conducted to ensure that the findings from the three sets of central analyses presented above were not affected by potential confounds including youth's age and gender, parents' gender, educational attainment, and birthplace, family type, and number of children. To this end, all the analyses were re-run without including these covariates. There were no meaningful changes in the significance or size of the findings as reported above, suggesting the robustness of these findings. Specifically, greater parental burnout was still predictive of 
Table 2 Moderation effects of emotion regulation on the links between parental burnout and youth's mental health

\begin{tabular}{|c|c|c|c|c|c|c|}
\hline & \multicolumn{3}{|c|}{$\begin{array}{l}\text { Predicting youth's depressive } \\
\text { symptoms }\end{array}$} & \multicolumn{3}{|c|}{$\begin{array}{l}\text { Predicting youth's anxiety } \\
\text { symptoms }\end{array}$} \\
\hline & $B$ & $S E$ & $\beta$ & $B$ & $S E$ & $\beta$ \\
\hline \multicolumn{7}{|c|}{ Model 1 (Cognitive reappraisal): } \\
\hline Child age & 0.16 & 0.11 & 0.06 & -0.04 & 0.10 & -0.02 \\
\hline Child gender & -0.03 & 0.04 & -0.03 & -0.08 & 0.04 & $-0.11^{*}$ \\
\hline Parent gender & 0.05 & 0.05 & 0.05 & 0.02 & 0.04 & 0.02 \\
\hline Parent education & -0.06 & 0.05 & -0.06 & -0.02 & 0.04 & -0.03 \\
\hline Parent birthplace & -0.03 & 0.04 & -0.04 & -0.03 & 0.04 & -0.05 \\
\hline Family type & 0.06 & 0.05 & 0.06 & 0.04 & 0.05 & 0.04 \\
\hline Number of children & 0.02 & 0.08 & 0.01 & 0.02 & 0.07 & 0.02 \\
\hline Prior adjustment & 0.50 & 0.04 & $0.54^{* * *}$ & 0.35 & 0.05 & $0.38^{* * *}$ \\
\hline Parental burnout & 0.13 & 0.05 & $0.13^{* *}$ & 0.14 & 0.04 & $0.18^{* * *}$ \\
\hline Cognitive reappraisal & -0.07 & 0.04 & $-0.09^{*}$ & -0.11 & 0.03 & $-0.17^{* *}$ \\
\hline Burnout $\times$ reappraisal & -0.10 & 0.05 & $-0.10^{*}$ & -0.11 & 0.04 & $-0.14^{* *}$ \\
\hline \multicolumn{7}{|c|}{ Model 2 (Expressive suppression): } \\
\hline Child age & 0.19 & 0.11 & 0.08 & 0.01 & 0.10 & 0.00 \\
\hline Child gender & -0.02 & 0.04 & -0.02 & -0.07 & 0.04 & -0.10 \\
\hline Parent gender & 0.05 & 0.05 & 0.05 & 0.00 & 0.04 & 0.00 \\
\hline Parent education & -0.07 & 0.05 & -0.07 & -0.03 & 0.04 & -0.04 \\
\hline Parent birthplace & -0.04 & 0.04 & -0.04 & -0.04 & 0.04 & -0.06 \\
\hline Family type & 0.07 & 0.05 & 0.06 & 0.04 & 0.05 & 0.05 \\
\hline Number of children & 0.01 & 0.08 & 0.01 & 0.01 & 0.07 & 0.01 \\
\hline Prior adjustment & 0.51 & 0.04 & $0.54^{* * *}$ & 0.36 & 0.05 & $0.39^{* * *}$ \\
\hline Parental burnout & 0.18 & 0.05 & $0.18^{* *}$ & 0.19 & 0.05 & $0.24^{* * *}$ \\
\hline Expressive suppression & -0.04 & 0.03 & -0.06 & -0.05 & 0.03 & -0.09 \\
\hline Burnout $\times$ suppression & -0.07 & 0.04 & -0.09 & -0.06 & 0.04 & -0.11 \\
\hline
\end{tabular}

For gender, $-1=$ male and $1=$ female. For parental education, $-1=$ less than a college degree and $1=$ college degree or higher. For parent birthplace, $-1=$ born in Shanghai, $1=$ not born in Shanghai. For family type, $-1=$ two-parent biological family, $1=$ other types

$* p<0.05, * * p<0.01, * * * p<0.001$ youth's greater depressive and anxiety symptoms over time, $\beta s>0.14, p s<0.01$. Moreover, sensitivity analyses regarding the mediation effect of parental autonomy support and the moderation effect of parents' cognitive reappraisal revealed similar findings as reported above.

\section{Discussion}

Parental burnout is a feeling of exhaustion in one's parental role resulting from enduring exposure to parenting stress (Mikolajczak et al., 2019). Given that parental burnout may be detrimental to child development (Mikolajczak et al., 2018; Mikolajczak et al., 2018), it is important to examine the longitudinal effects of parental burnout on youth's adjustment, investigate the underlying mechanisms, and identify protective factors that buffer such negative impacts. Using a two-wave longitudinal approach, the current research found that parental burnout was predictive of youth's greater depressive and anxiety symptoms over two months, and these effects were partially mediated by less autonomy-supportive parenting. Notably, parental burnout did not have negative consequences on autonomysupportive parenting or youth's mental health when parents used greater cognitive reappraisal to regulate negative emotions, suggesting that effective emotion regulation can be a protective factor against parental burnout.

\section{The Effects of Parental Burnout on Youth's Mental Health}

In line with the hypotheses, parental burnout predicted youth's greater depressive and anxiety symptoms over the two-month period, controlling for youth's prior depressive/ anxiety symptoms as well as other covariates. These results are consistent with past research that suggests longitudinal 
Fig. 3 The effects of parental burnout on youth's depressive symptoms (Panel A) and anxiety (Panel B) were moderated by parents' use of cognitive reappraisal in emotion regulation. Low (or high) cognitive reappraisal is $1 \mathrm{SD}$ below (or above) the mean. The numbers in parentheses are unstandardized simple slopes. ${ }^{* * *} p<0.001$. ns not significant
Panel A

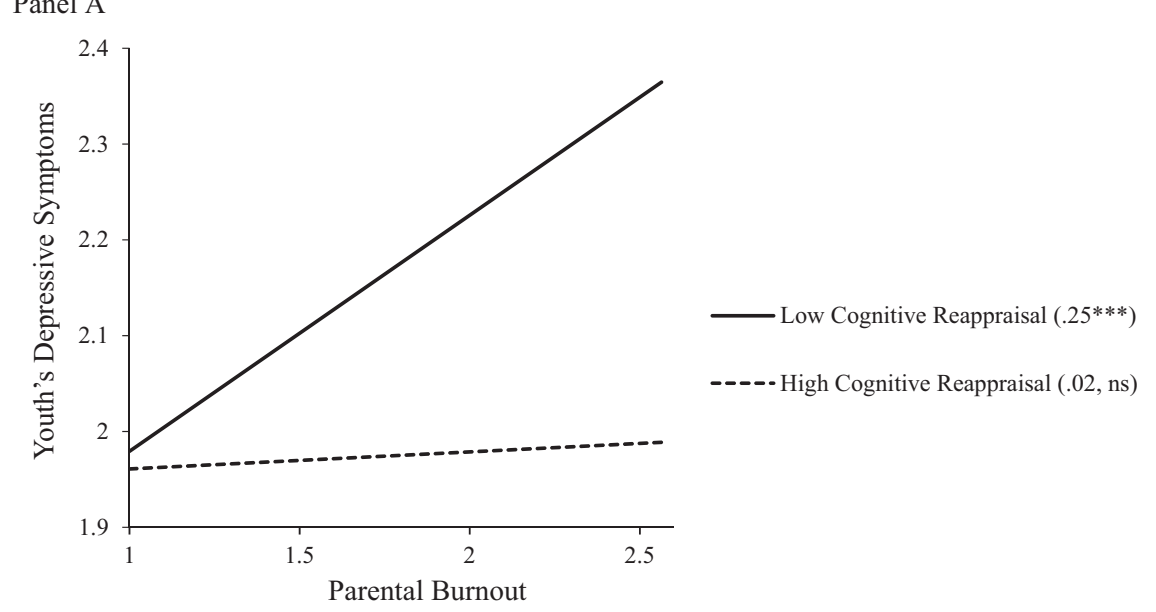

Panel B
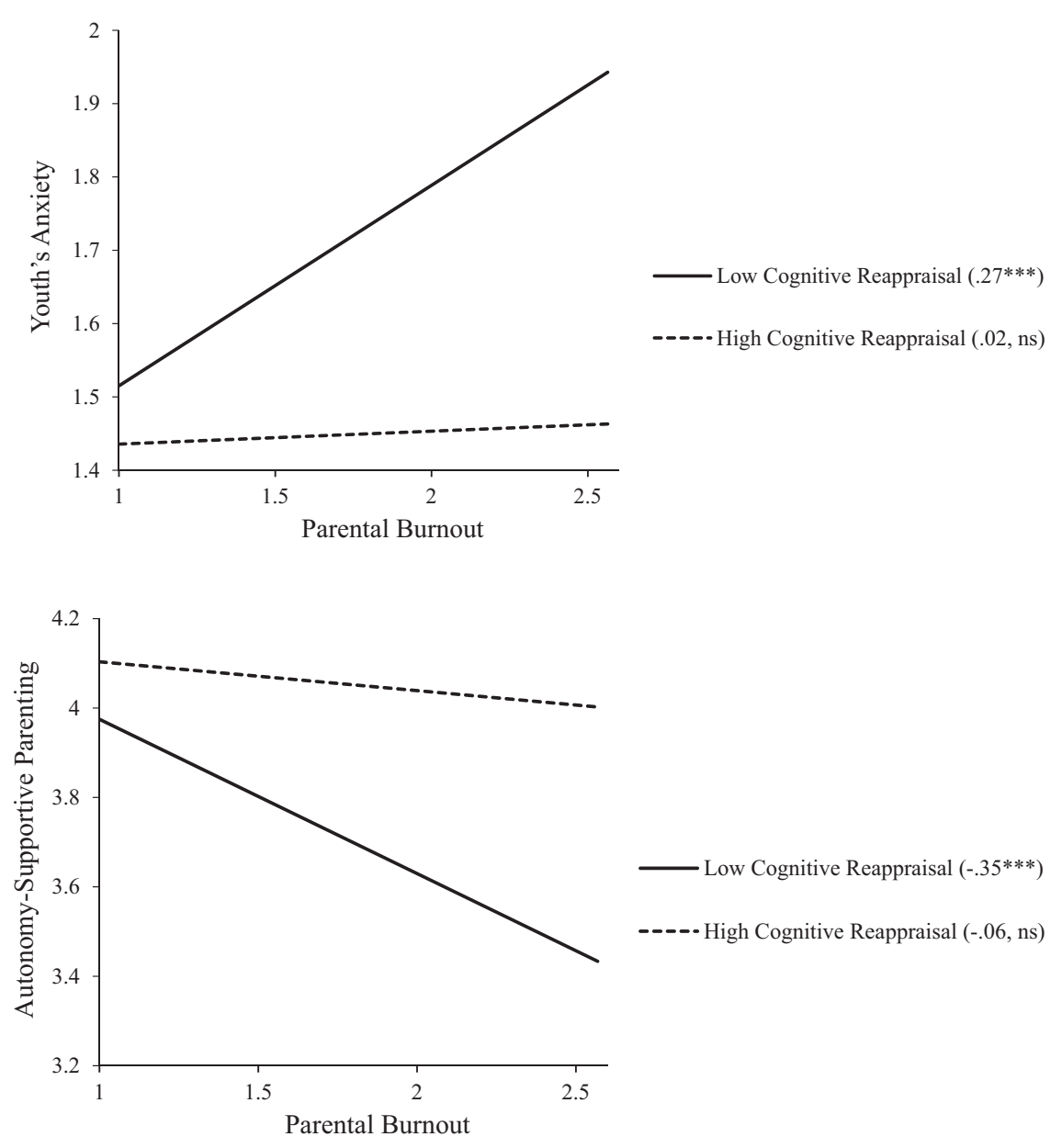

Fig. 4 The associations between parental burnout and autonomysupportive parenting were moderated by parents' use of cognitive reappraisal in emotion regulation. Note. Results with parent-reported autonomysupportive parenting were presented in the figure. Low (or high) cognitive reappraisal is $1 \mathrm{SD}$ below (or above) the mean. The numbers in parentheses are unstandardized simple slopes. ${ }^{* * *} p<0.001$. ns not significant associations between parents' stress level and youth's mental health (Bakoula et al., 2009). Moreover, the findings are in line with a recent study that suggests concurrent associations between parents' caregiver burden (e.g., feeling exhausted and incapable in one's caregiver responsibilities) and children's distress (e.g., feeling nervous and stressed)
(Russell et al., 2020). Importantly, these findings are the first to show longitudinal impacts of parental burnout on youth's psychological adjustment. The results highlight the detrimental role of parental burnout in adolescent development, such that it may have negative consequences on youth's mental health in a short period of time. 


\section{The Mediating Role of Autonomy-Supportive Parenting}

The longitudinal effects of parental burnout on youth's greater depressive and anxiety symptoms were partially mediated by less autonomy-supportive parenting. Youthreported and parent-reported autonomy support showed similar results in the mediation models, such that youthreported and parent-reported autonomy support both mediated the longitudinal effects of parental burnout on youth's mental health. The links between parental burnout and autonomy-supportive parenting are in congruence with existing literature on dampened parental autonomy support under stress (Grolnick, 2009; Wuyts et al., 2017). Prior research suggests that, in stressful environments, parents are less autonomy-supportive toward their children both verbally and non-verbally (e.g., give children less feedback/ encouragement and take over their children's task; Grolnick et al., 2002). In a similar vein, when parents are experiencing burnout, the feeling of exhaustion may make them less willing to consider their children's point of view and thus provide less autonomy support to their children. Although prior research has shown that parental burnout may result in parental neglect and violence (Mikolajczak et al., 2018; Mikolajczak et al., 2019), the consequence of parental burnout on autonomy support has been understudied. The results show that, as a potential consequence of parental burnout, the lack of autonomy support may have undesirable effects on youth's mental health in a relatively short period of time. This also adds an important piece of empirical evidence to self-determination theory, which suggests that autonomy is a basic psychological need, such that the lack of autonomy may induce mental health problems (Ryan \& Deci, 2000, 2006). Taken together, the current study suggests the possible underlying mechanism through which parental burnout may play a role in youth's mental health.

\section{The Moderating Role of Parents' Cognitive Reappraisal}

Parents' use of cognitive reappraisal to regulate emotions played a moderating role in the effects of parental burnout on youth's mental health and parents' parenting practices. In line with the hypotheses, cognitive reappraisal moderated the longitudinal associations between parental burnout and youth's depressive/anxiety symptoms. Moreover, cognitive reappraisal moderated the association between parental burnout and parent-reported, but not youth-reported, autonomy support. This finding points out potential parent-child discrepancy in reporting parenting practice. With regard to the link between parental burnout and parenting practice, the buffering effect of cognitive reappraisal may not be immediately perceived by youth, such that the association between parental burnout and youth-perceived autonomy support was not moderated by parents' cognitive reappraisal.

In families that parents reported low cognitive reappraisal, parental burnout was related to less autonomysupportive parenting, which was predictive of youth's greater depressive and anxiety symptoms over time. However, in families that parents reported high cognitive reappraisal, parental burnout was not negatively associated with autonomy-supportive parenting nor youth's mental health. This suggests that parents' use of cognitive reappraisal buffers the negative effects of parental burnout on parenting practices and youth's mental health. These results are in line with prior research that suggests the protecting role of cognitive reappraisal in response to parenting stress (DeaterDeckard et al., 2016). With greater use of cognitive reappraisal, parents may be more likely to reply on problemfocused coping instead of avoidance in response to parental burnout, and such problem-focused coping strategies are typically effective in coping with stress (Bartley \& Roesch, 2011). In this case, parents may be able to successfully cope with parental burnout, and thus become less affected by it. Therefore, the current findings highlight the role of parents' cognitive reappraisal as an important protective factor against parental burnout.

In contrast, parents' use of expressive suppression to regulate emotions did not buffer the negative effects of parental burnout on youth's mental health. Results are consistent with past studies which show that expressive suppression (vs. cognitive reappraisal) is less effective in regulating negative emotions (e.g., Dryman \& Heimberg, 2018; Johnson et al., 2016). The current research suggests that suppression is not an effective protective factor against parental burnout. Even though parents may attempt to inhibit the expression of their negative emotions for a good intention, such effort may be ineffective in protecting their children's psychological well-being from parental burnout. It is important to note that, although past studies have demonstrated the negative impacts of expressive suppression on individuals' mental health in Western cultures (Butler et al., 2003; Haga et al., 2009), the current research did not find the similar pattern (i.e., parents' expressive suppression was not correlated with youth's mental health at either wave as shown in Table 1 and did not exacerbate the negative effects of parental burnout on youth's mental health as shown in Table 2). In Chinese culture, emotional control and restraint are considered as merits and are still prevalent in contemporary China (Yik, 2010). In this case, both expressive suppression and cognitive reappraisal may be common approaches of emotion regulation in the Chinese context. Consistent with prior studies on emotion regulation in Asian youth (Chen et al., 2020; Yeh et al., 2017), the current study found that expressive suppression 
was positively correlated with cognitive reappraisal. Moreover, past research suggests that expressive suppression may be less harmful to psychological well-being in the Chinese cultural context compared to in the Western cultural context (Soto et al., 2011). In line with this research, the results suggest that, although parents' expressive suppression does not buffer the negative effects of parental burnout on youth's mental health, it also does not exacerbate such negative effects.

\section{Theoretical and Practical Implications}

The findings of the current research have important theoretical and practical implications. There have been vast interests in how parental burnout may affect child development (Cheng et al., 2020; Mikolajczak et al., 2019). To our knowledge, the current research was the first to investigate the longitudinal consequences of parental burnout on adolescents' adjustment with attention to the underlying mechanisms. The current research provides important empirical evidence of how parental burnout may affect adolescent development through parenting practices. With regard to practical implications, the findings provide initial evidence that can be used in future interventions aiming at reducing the negative impacts of parental burnout. The results suggest that a high level of parents' cognitive reappraisal can buffer the negative effects of parental burnout on parenting practices and adolescent development. Therefore, interventions that seek to improve parents' emotion regulation skills (e.g., interventions that foster the use of cognitive reappraisal to regulate emotions, Kivity \& Huppert, 2016; Rodriguez et al., 2020) may be effective in alleviating the maladaptive consequences of parental burnout, and such interventions may be particularly useful during stressful times such as the current pandemic.

\section{Limitations and Future Directions}

There are several limitations in the current study that point to directions for future research. First, the current study focused on youth's mental health as the outcome, and thus whether parental burnout has similar impacts on other aspects of adolescent development is yet unknown. Past studies suggest that parenting stress is longitudinally associated with children's behavioral problems (e.g., more conduct problems and less prosocial behaviors; Megahead \& Deater-Deckard, 2017; Neece et al., 2012). Given that parental burnout is resulted from enduring exposure to parenting stress (Roskam et al., 2017), it may also influence adolescent development in the behavioral domain such as conduct disorder and risk-taking behavior. Therefore, a key direction for future research is to examine the effects of parental burnout on youth's behavioral development over time.
Second, the current research followed parent-child dyads over a short period of time (i.e., two months) during the COVID-19 pandemic outbreak, which leaves an open question regarding whether parental burnout has a similar effect on youth's mental health during a normal time or/and over a longer period. Scholars have called more attention to parental burnout during COVID-19 (Griffith, 2020), because parents may suffer from a number of parenting-related stressors such as children's limited access to education due to school closures and concerns about children's health (Fontanesi et al., 2020; Russell et al., 2020). Given the heightened vulnerability of mental health during COVID-19 (Alonzi et al., 2020), youth's mental health may be less stable during a pandemic compared to during a normal time. Therefore, parental burnout may be more likely to play a role in youth's mental health within this short period of time (i.e., two months) during the pandemic. It is possible that, during a normal time, parental burnout may have similar effects on youth's mental health over a longer period, but not over a short period of time. Future studies can employ longitudinal designs over multiple timepoints to examine both the short-term and long-term impacts of parental burnout on youth's mental health during a normal time. Moreover, because the current study only measured parental burnout at Wave 1, it remains unknown whether parental burnout has changed over time. Although this is not the focus of the current research, it would be informative to investigate the stability of parental burnout over time. Future studies can assess parental burnout for two or more times to examine whether parental burnout is stable over time.

Finally, the current study focused on parental burnout and adolescent development in Chinese families, and thus it remains unclear whether parental burnout has similar effects on youth's mental health in other countries. Because the relations between parental burnout and negative family characteristics (e.g., family disorganization, parental neglect, and co-parenting disengagement) are fairly consistent across cultures (Mikolajczak et al., 2019; Furutani et al., 2020), it is highly likely that parental burnout also has similar effects on youth's mental health in other countries. Therefore, it is important for future research to investigate whether parental burnout plays a similar or different role in youth's adjustment across countries and cultures.

\section{Conclusion}

Under the influence of enduring exposure to parenting stress, parents may be at risk of parental burnout, which can have an impact on youth's mental health. However, no prior research has examined how parental burnout affects youth's mental health over time and identified protective factors that may buffer such negative effects. Using a longitudinal approach, the current study found that parental burnout was 
predictive of Chinese youth's greater depressive and anxiety symptoms over time, and the effects were mediated, in part, by less autonomy-supportive parenting. Importantly, the effects of parental burnout on autonomy support and youth's mental health were buffered by parents' use of cognitive reappraisal to regulate negative emotions. Taken together, the current research demonstrates the longitudinal effects of parental burnout on youth's mental health through parenting practices and highlights the protective effects of emotion regulation. Interventions and policy recommendations aiming at reducing the detrimental impacts of parental burnout should consider the buffering role of effective emotion regulation.

Acknowledgements We would like to thank all the families participating in this study.

Authors' Contributions B.Y. was involved in designing the measures, developed the hypotheses motivating the data analyses, performed the statistical analyses, interpreted the results, and drafted the manuscript; B.C. conceived of the study, participated in the study design, performed data collection, interpreted the results, and revised the manuscript; Y.Q. conceived of the study, participated in the study design, oversaw the data analysis, interpreted the results, and drafted the manuscript; Y.Z. participated in the study design, performed data collection, and revised the manuscript. All authors read and approved the final manuscript.

Data Sharing and Declaration The datasets generated and/or analyzed during the current study are not publicly available but are available from the corresponding author on reasonable request.

\section{Compliance with Ethical Standards}

Conflict of Interest The authors declare no competing interests.

Ethical Approval All procedures performed in studies involving human participants were in accordance with the ethical standards of the institutional and/or national research committee and with the 1964 Helsinki declaration and its later amendments or comparable ethical standards.

Informed Consent Consent forms were obtained from all participants included in the study.

Publisher's note Springer Nature remains neutral with regard to jurisdictional claims in published maps and institutional affiliations.

\section{References}

Alonzi, S., La Torre, A., \& Silverstein, M. W. (2020). The psychological impact of preexisting mental and physical health conditions during the COVID-19 pandemic. Psychological Trauma: Theory, Research, Practice, and Policy. https://doi.org/10.1037/ tra0000840

Angold, A., Costello, E. J., Messer, S. C., Pickles, A., Winder, F., \& Silver, D. (1995). Development of a short questionnaire for use in epidemiological studies of depression in children and adolescents.
International Journal of Methods in Psychiatric Research, 5, $1-13$.

Bakoula, C., Kolaitis, G., Veltsista, A., Gika, A., \& Chrousos, G. P. (2009). Parental stress affects the emotions and behaviour of children up to adolescence: A Greek prospective, longitudinal study. Stress, 12(6), 486-498. https://doi.org/10.3109/ 10253890802645041 .

Barber, B. K., Stolz, H. E., \& Olsen, J. A. (2005). Parental support, psychological control, and behavioral control: Assessing relevance across time, culture, and method. Monographs of the Society for Research in Child Development, i-147. https://doi.org/ 10.1111/j.1540-5834.2005.00365.x

Bartley, C. E., \& Roesch, S. C. (2011). Coping with daily stress: The role of conscientiousness. Personality and Individual Differences, 50(1), 79-83. https://doi.org/10.1016/j.paid.2010.08.027.

Brennan, P. A., Hammen, C., Katz, A. R., \& Le Brocque, R. M. (2002). Maternal depression, paternal psychopathology, and adolescent diagnostic outcomes. Journal of Consulting and Clinical Psychology, 70(5), 1075-1085. https://doi.org/10.1037/ 0022-006X.70.5.1075.

Butler, E. A., Egloff, B., Wlhelm, F. H., Smith, N. C., Erickson, E. A., \& Gross, J. J. (2003). The social consequences of expressive suppression. Emotion, 3(1), 48-67. https://doi.org/10.1037/15283542.3.1.48.

Chen, J., Zhang, C., Wang, Y., \& Xu, W. (2020). A longitudinal study of inferiority impacting on aggression among college students: the mediation role of cognitive reappraisal and expression suppression. Personality and Individual Differences, 157, 109839 https://doi.org/10.1016/j.paid.2020.109839.

Cheng, H., Wang, W., Wang, S., Li, Y., Liu, X., \& Li, Y. (2020). Validation of a Chinese version of the parental burnout assessment. Frontiers in Psychology, 11, 321 https://doi.org/10.3389/ fpsyg.2020.00321.

Cheung, C. S., Pomerantz, E. M., Wang, M., \& Qu, Y. (2016). Controlling and autonomy-supportive parenting in the United States and China: Beyond children's reports. Child Development, 87(6), 1992-2007. https://doi.org/10.1111/cdev.12567.

Deater-Deckard, K. (1998). Parenting stress and child adjustment: Some old hypotheses and new questions. Clinical psychology: Science and Practice, 5(3), 314-332. https://doi.org/10.1111/j. 1468-2850.1998.tb00152.x.

Deater-Deckard, K., Li, M., \& Bell, M. A. (2016). Multifaceted emotion regulation, stress and affect in mothers of young children. Cognition and Emotion, 30(3), 444-457. https://doi.org/10. 1080/02699931.2015.1013087.

Dryman, M. T., \& Heimberg, R. G. (2018). Emotion regulation in social anxiety and depression: a systematic review of expressive suppression and cognitive reappraisal. Clinical Psychology Review, 65, 17-42. https://doi.org/10.1016/j.cpr.2018.07.004.

Fontanesi, L., Marchetti, D., Mazza, C., Di Giandomenico, S., Roma, P. \& Verrocchio, M. C. (2020). The effect of the COVID19 lockdown on parents: A call to adopt urgent measures. Psychological Trauma: Theory, Research, Practice, and Policy, 12(1), 79-81. https://doi.org/10.1037/tra0000672.

Frasquilho, D., de Matos, M. G., Marques, A., Neville, F. G., Gaspar, T., \& Caldas-de-Almeida, J. M. (2016). Unemployment, parental distress and youth emotional well-being: The moderation roles of parent-youth relationship and financial deprivation. Child Psychiatry \& Human Development, 47(5), 751-758. https://doi.org/ 10.1007/s10578-015-0610-7.

Furutani, K., Kawamoto, T., Alimardani, M., \& Nakashima, K. I. (2020). Exhausted parents in Japan: preliminary validation of the Japanese version of the Parental Burnout Assessment. New Directions for Child and Adolescent Development, 174, 33-49. https://doi.org/10.1002/cad.20371. 
Griffith, A. K. (2020). Parental burnout and child maltreatment during the COVID-19 pandemic. Journal of Family Violence, 1-7. https://doi.org/10.1007/s10896-020-00172-2.

Grolnick, W. S. (2009). The role of parents in facilitating autonomous self-regulation for education. Theory and Research in Education, 7(2), 164-173. https://doi.org/10.1177/2F1477878509104321.

Grolnick, W. S., Gurland, S. T., DeCourcey, W., \& Jacob, K. (2002). Antecedents and consequences of mothers' autonomy support: An experimental investigation. Developmental Psychology, 38 (1), 143-155. https://doi.org/10.1037/0012-1649.38.1.143.

Gross, J. J., \& John, O. P. (2003). Individual differences in two emotion regulation processes: Implications for affect, relationships, and well-being. Journal of Personality and Social Psychology, 85(2), 348-362. https://doi.org/10.1037/0022-3514.85. 2.348

Haga, S. M., Kraft, P., \& Corby, E. K. (2009). Emotion regulation: Antecedents and well-being outcomes of cognitive reappraisal and expressive suppression in cross-cultural samples. Journal of Happiness Studies, 10(3), 271-291. https://doi.org/10.1007/ s10902-007-9080-3.

John, O. P., \& Gross, J. J. (2004). Healthy and unhealthy emotion regulation: personality processes, individual differences, and life span development. Journal of Personality, 72(6), 1301-1334. https://doi.org/10.1111/j.1467-6494.2004.00298.x.

Johnson, J., O’Connor, D. B., Jones, C., Jackson, C., Hughes, G. J., \& Ferguson, E. (2016). Reappraisal buffers the association between stress and negative mood measured over 14 days: Implications for understanding psychological resilience. European Journal of Personality, 30(6), 608-617. https://doi.org/10.1002/per.2080.

Kao, K., Tuladhar, C. T., Meyer, J. S., \& Tarullo, A. R. (2019). Emotion regulation moderates the association between parent and child hair cortisol concentrations. Developmental Psychobiology, 61(7), 1064-1078. https://doi.org/10.1002/dev.21850.

Kivity, Y., \& Huppert, J. D. (2016). Does cognitive reappraisal reduce anxiety? A daily diary study of a micro-intervention with individuals with high social anxiety. Journal of Consulting and Clinical Psychology, 84(3), 269-283. https://doi.org/10.1037/ ccp0000075.

Koepke, S., \& Denissen, J. J. (2012). Dynamics of identity development and separation-individuation in parent-child relationships during adolescence and emerging adulthood - A conceptual integration. Developmental Review, 32(1), 67-88. https://doi.org/ 10.1016/j.dr.2012.01.001.

Lee, F. S., Heimer, H., Giedd, J. N., Lein, E. S., Šestan, N., Weinberger, D. R., \& Casey, B. J. (2014). Adolescent mental health-Opportunity and obligation. Science, 346(6209), 547-549. https://doi.org/10.1126/science.1260497.

Leiter, M. P., Maslach, C., \& Frame, K. (2014). Burnout. The Encyclopedia of Clinical Psychology, 1-7. https://doi.org/10.1002/ 9781118625392.wbecp142

Little, R. J. (1988). A test of missing completely at random for multivariate data with missing values. Journal of the American statistical Association, 83(404), 1198-1202. https://doi.org/10.1080/ 01621459.1988.10478722.

Marbell-Pierre, K. N., Grolnick, W. S., Stewart, A. L., \& RafteryHelmer, J. N. (2019). Parental autonomy support in two cultures: the moderating effects of adolescents' self-construals. Child Development, 90(3), 825-845. https://doi.org/10.1111/cdev. 12947.

Maslach, C., \& Leiter, M. P. (2016). Understanding the burnout experience: recent research and its implications for psychiatry. World Psychiatry, 15(2), 103-111. https://doi.org/10.1002/wps. 20311.

McPartland, J. M., \& Epstein, J. L. (1977). Open schools and achievement: Extended tests of a finding of no relationship.
Sociology of Education, 133-144. https://doi.org/10.2307/ 2112375

Megahead, H. A., \& Deater-Deckard, K. (2017). Parenting stress and foster children's adjustment in an Egyptian context. Journal of Child and Family Studies, 26(8), 2266-2275. https://doi.org/10. 1007/s10826-017-0736-2.

Mikolajczak, M., Brianda, M. E., Avalosse, H., \& Roskam, I. (2018). Consequences of parental burnout: Its specific effect on child neglect and violence. Child Abuse \& Neglect, 80, 134-145. https://doi.org/10.1016/j.chiabu.2018.03.025.

Mikolajczak, M., Gross, J. J., \& Roskam, I. (2019). Parental burnout: What is it, and why does it matter? Clinical Psychological Science, 7(6), 1319-1329. 10.1177\%2F2167702619858430.

Mikolajczak, M., Raes, M. E., Avalosse, H., \& Roskam, I. (2018). Exhausted parents: Sociodemographic, child-related, parent-related, parenting and family-functioning correlates of parental burnout. Journal of Child and Family Studies, 27(2), 602-614. https://doi.org/10.1007/s10826-017-0892-4.

Muthén, L. K., \& Muthén, B. O. (2017). Mplus user's guide. Los Angeles, CA.

Neece, C. L., Green, S. A., \& Baker, B. L. (2012). Parenting stress and child behavior problems: A transactional relationship across time. American Journal on Intellectual and Developmental Disabilities, 117(1), 48-66. https://doi.org/10.1352/1944-7558-117. 1.48 .

Orben, A., \& Przybylski, A. K. (2019). The association between adolescent well-being and digital technology use. Nature Human Behaviour, 3(2), 173-182. https://doi.org/10.1038/s41562-0180506-1.

Pereira, A. I., Barros, L., Mendonça, D., \& Muris, P. (2014). The relationships among parental anxiety, parenting, and children's anxiety: The mediating effects of children's cognitive vulnerabilities. Journal of Child and Family Studies, 23(2), 399-409. https://doi.org/10.1007/s10826-013-9767-5.

Qu, Y., Rompilla, D. B., Wang, Q., \& Ng, F. F. Y. (2020). Youth's negative stereotypes of teen emotionality: reciprocal relations with emotional functioning in Hong Kong and Mainland China. Journal of Youth and Adolescence, 49(10), 2003-2019. https:// doi.org/10.1007/s10964-020-01303-0.

Robbins, R. J. (1994). An assessment of perceptions of parental autonomy support and control: Child and parent correlates. Unpublished doctoral dissertation, University of Rochester.

Rodriguez, L. M., Lee, K. D., Onufrak, J., Dell, J. B., Quist, M., Drake, H. P., \& Bryan, J. (2020). Effects of a brief interpersonal conflict cognitive reappraisal intervention on improvements in access to emotion regulation strategies and depressive symptoms in college students. Psychology \& Health, 1-21. https://doi.org/ 10.1080/08870446.2019.1711090

Roskam, I., Aguiar, J., Akgun, E., Arikan, G., Artavia, M., Avalosse, H., ... \& Mikolajczak, M. (2021). Parental burnout around the globe: a 42-country study. Affective Science, 2, 58-79. https://doi. org/10.1007/s42761-020-00028-4

Roskam, I., Brianda, M. E., \& Mikolajczak, M. (2018). A step forward in the conceptualization and measurement of parental burnout: the parental burnout assessment (PBA). Frontiers in Psychology, 9, 758. https://doi.org/10.3389/fpsyg.2018.00758

Roskam, I., Raes, M. E., \& Mikolajczak, M. (2017). Exhausted parents: development and preliminary validation of the parental burnout inventory. Frontiers in Psychology, 8, 163 https://doi. org/10.3389/fpsyg.2017.00163.

Russell, B. S., Hutchison, M., Tambling, R., Tomkunas, A. J., \& Horton, A. L. (2020). Initial challenges of caregiving during COVID-19: Caregiver burden, mental health, and the parent-child relationship. Child Psychiatry \& Human Development, 51(5), 671-682. https://doi.org/10.1007/s10578-020-01037-x. 
Rutherford, H. J., Wallace, N. S., Laurent, H. K., \& Mayes, L. C. (2015). Emotion regulation in parenthood. Developmental Review, 36, 1-14. https://doi.org/10.1016/j.dr.2014.12.008.

Ryan, R. M. \& Deci, E. L. (2000). Self-determination theory and the facilitation of intrinsic motivation, social development, and wellbeing. American Psychologist, 55(1), 68-78. https://doi.org/10. 1037/110003-066X.55.1.68.

Ryan, R. M., \& Deci, E. L. (2006). Self-regulation and the problem of human autonomy: Does psychology need choice, self-determination, and will? Journal of Personality, 74(6), 1557-1586. https://doi.org/10.1111/j.1467-6494.2006.00420.x.

Ryan, R. M., \& Deci, E. L. (2017). Self-determination theory: Basic psychological needs in motivation, development, and wellness. Guilford Publications.

Shahane, A. D., Lopez, R. B., \& Denny, B. T. (2019). Implicit reappraisal as an emotional buffer: reappraisal-related neural activity moderates the relationship between inattention and perceived stress during exposure to negative stimuli. Cognitive, Affective, \& Behavioral Neuroscience, 19(2), 355-365. https://doi.org/10. 3758/s13415-018-00676-x.

Soenens, B., Vansteenkiste, M., \& Van Petegem, S. (2015). Let us not throw out the baby with the bathwater: Applying the principle of universalism without uniformity to autonomy-supportive and controlling parenting. Child Development Perspectives, 9(1), 44-49. https://doi.org/10.1111/cdep.12103.

Soenens, B., Vansteenkiste, M., Lens, W., Luyckx, K., Goossens, L., Beyers, W., \& Ryan, R. M. (2007). Conceptualizing parental autonomy support: Adolescent perceptions of promotion of independence versus promotion of volitional functioning. Developmental Psychology, 43(3), 633-646. https://doi.org/10. 1037/0012-1649.43.3.633.

Sorkkila, M., \& Aunola, K. (2020). Risk factors for parental burnout among Finnish parents: the role of socially prescribed perfectionism. Journal of Child and Family Studies, 29(3), 648-659. https://doi.org/10.1007/s10826-019-01607-1.

Soto, J. A., Perez, C. R., Kim, Y. H., Lee, E. A., \& Minnick, M. R. (2011). Is expressive suppression always associated with poorer psychological functioning? A cross-cultural comparison between European Americans and Hong Kong Chinese. Emotion, 11(6), 1450-1455. https://doi.org/10.1037/a0023340.

Speer, M. E., \& Delgado, M. R. (2017). Reminiscing about positive memories buffers acute stress responses. Nature Human Behaviour, 1(5), 1-9. https://doi.org/10.1038/s41562-017-0093.

Spitzer, R. L., Kroenke, K., Williams, J. B., \& Löwe, B. (2006). A brief measure for assessing generalized anxiety disorder: The GAD-7. Archives of Internal Medicine, 166(10), 1092-1097. https://doi.org/10.1001/archinte.166.10.1092.

Steinberg, L., Lamborn, S. D., Dornbusch, S. M., \& Darling, N. (1992). Impact of parenting practices on adolescent achievement: Authoritative parenting, school involvement, and encouragement to succeed. Child Development, 63(5), 1266-1281. https://doi. org/10.1111/j.1467-8624.1992.tb01694.x.

van der Kaap-Deeder, J., Vansteenkiste, M., Soenens, B., \& Mabbe, E. (2017). Children's daily well-being: The role of mothers', teachers', and siblings' autonomy support and psychological control.
Developmental Psychology, 53(2), 237-251. https://doi.org/10. 1037/dev0000218.

Wang, Q., Pomerantz, E. M., \& Chen, H. (2007). The role of parents' control in early adolescents' psychological functioning: a longitudinal investigation in the United States and China. Child Development, 78(5), 1592-1610. https://doi.org/10.1111/j.14678624.2007.01085.x.

Wang, Y., Chen, R., \& Zhang, L. (2018). Reliability and validity of generalized anxiety scale-7 in inpatients in Chinese general hospital. The Journal of Clinical Psychiatry, 28, 168-171. https:// doi.org/10.3969/j.issn.1005-3220.2018.03.007.

Wuyts, D., Soenens, B., Vansteenkiste, M., Van Petegem, S., \& Brenning, K. (2017). The role of separation anxiety in mothers' use of autonomy support: An observational study. Journal of Child and Family Studies, 26(7), 1949-1957. https://doi.org/10. 1007/s10826-017-0707-7.

Yeh, K. H., Bedford, O., Wu, C. W., Wang, S. Y., \& Yen, N. S. (2017). Suppression benefits boys in Taiwan: The relation between gender, emotional regulation strategy, and mental health. Frontiers in psychology, 8, 135 https://doi.org/10.3389/fpsyg.2017.00135.

Yik, M. (2010). How unique is Chinese emotion? In M. H. Bond (Ed.), The Oxford handbook of Chinese psychology (pp. 205-220). New York, NY: Oxford University Press.

Beiming Yang is a Ph.D. student in Human Development and Social Policy at Northwestern University. His major research interests include parenting, children's motivation, and emotional well-being in different cultures.

Bin-Bin Chen is an associate professor in the Department of Psychology at Fudan University. His major research interests include parenting, sibling relationships, children and adolescents' socioemotional development.

Yang Qu is an assistant professor in the School of Education and Social Policy at Northwestern University. His research focuses on the role of sociocultural contexts in adolescents' academic, social, and emotional development.

Yuanfei Zhu is a Ph.D. student in the School of Psychology and Cognitive Science at East China Normal University. He also serves as a vice director at Shanghai Teacher Training Center. His major research interests include teacher training, teaching for creativity and child development. 